\title{
World irradiation facilities for silicon detectors
}

\author{
Vladimir Cindro \\ Jožef Stefan Institute \\ Jamova 39, 1000 Ljubljana, Slovenia \\ E-mail: vladimir.cindro@ijs.si
}

\begin{abstract}
Several irradiation facilities are used for studies of radiation hardness of silicon detectors. Variety of particles and energies allow better understanding of damage mechanism. Future upgrades of experiments will demand even an order of magnitude higher fluences in irradiation tests. An overview of most suitable and accessible irradiation facilities is given in this paper.
\end{abstract}

The 23rd International Workshop on Vertex Detectors

15-19 September 2014

Macha Lake, The Czech Republic

\section{Introduction}

The development of radiation hard semiconductor detectors is a wide spread task in which many groups from all over the world joined their effort to contribute to the common goal: to build experiments with detectors components that will survive a harsh radiation environment over the lifetime of experiment. The upgrade scenario of the LHC to a luminosity of $10^{35} \mathrm{~cm}^{-2}$ represents a challenge for developing sensors as well as for their tests after irradiations under realistic conditions. Fluences of fast hadrons above $10^{16} \mathrm{~cm}^{-2}$ are expected in the most exposed regions of detectors. Spectra of particles cover a wide range with energies up to tens of $\mathrm{GeV}$ [1]. Radiation damage will be mainly caused by hadrons, therefore irradiation facilities providing these particles are playing important role in the studies of damage effects. Irradiation facilities cannot exactly reproduce the spectra of particles at high energy experiments therefore it is important to make irradiations with different particles and energies to understand the damage mechanism in detectors.

\section{Radiation damage}

Atomic displacement and ionization are caused by energy transfer from radiation to material and serious degradation effects can thereby be caused. Increase of leakage current [2], change of effective dopant concentration [3] and increased trapping of drifting charge carriers [4] are the mostly recognized effects of atomic displacement, also called bulk damage. Trapped charge on deep traps in insulator and single event phenomena can be caused by ionization. Non Ionizing Energy Loss (NIEL) scaling to the energy loss of $1 \mathrm{MeV}$ neutrons is common concept used to compare the bulk damage of various particles with different energies. However this hypothesis has proved to be valid only for leakage current caused by hadrons [2], but it is not valid for trapping and the effective dopant concentration. For example, the trapping damage constant is higher for neutrons than for protons after the same NIEL [3], while higher oxygen concentration in silicon helps to reduce the change of the effective dopant concentration in float zone material after proton radiation, while it has little or no effect after neutron irradiation [5]. Even more dramatic are differences between neutron and proton induced damage in $n$ type magnetic Chocralski material [6]. Difference can be explained by the different distribution of interstitials and vacancies after charged or 
neutron hadron irradiation.

The relative composition of the irradiating particles will also vary across detectors, with pions, protons and neutrons being responsible for most of the bulk damage in material. Therefore it is of vital importance to understand the damage mechanism and perform irradiations with different kinds of particles having different energies.

\section{CERN Irradiation facility}

The CERN Proton Synchrotron (PS) [7,8] accelerates protons to a momentum of 24 $\mathrm{GeVc}^{-1}$. It has been a valuable source of high intensity proton beams for many irradiations in recent years. The complete reconstruction of the proton irradiation facility in the PS East Area (IRRAD) was completed in autumn 2014. In this new facility (Fig.1), located on the T8 beamline, higher beam intensities than in the past will become possible. Moreover, in the new facility, the beam spot can be varied from $5 \times 5 \mathrm{~mm}^{2}$ to $20 \times 20 \mathrm{~mm}^{2}$ (FWHM) with a maximum of 6 spills per PS super cycle, each spill containing about $510^{11}$ protons [9]. A cooling system can cool samples down to $-20^{\circ} \mathrm{C}$, while remotely controlled moving tables allow irradiation of larger samples. Setup for irradiations at cryogenic conditions, beam profile monitoring and dosimetry with foils and $10 \%$ accuracy are also available. The damage has been measured as 0.62 for $24 \mathrm{GeVc}^{-1}$ protons [10]. With twice as frequent spills foreseen in the future, irradiations at $10^{17} \mathrm{pcm}^{-2}$ with a focused beam ( $5 \mathrm{~mm}$ FWHM) will be possible in about 4 days.

The mixed field CHARM Facility [11] is located behind the IRRAD facility. The goal of this complex project is to provide irradiations with different hadron types having broad spectra in the $\mathrm{MeV}$ to several $\mathrm{GeV}$ region simulating the environment in the LHC tunnel and for space applications. In CHARM, the PS proton beam will hit various targets and movable shielding absorbers can be applied. The maximum flux of high energy hadrons will be about $10^{10} \mathrm{~cm}^{-2} \mathrm{~h}^{-2}$.

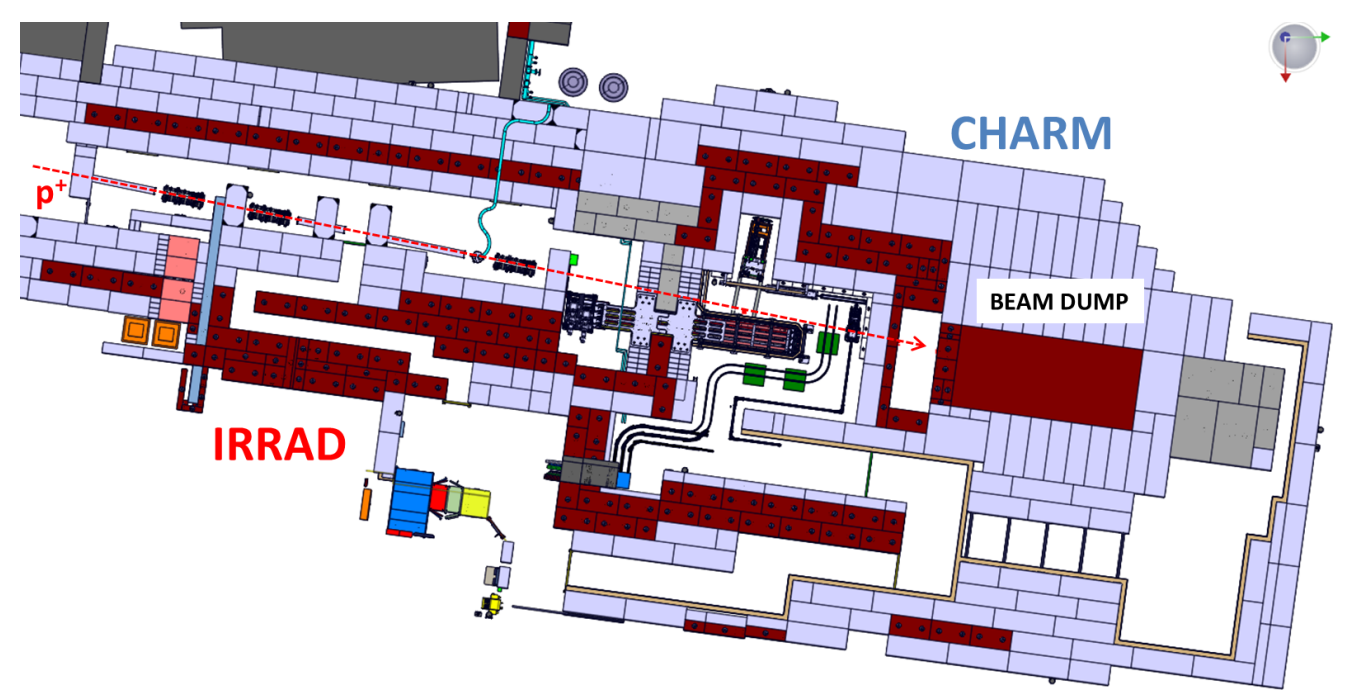

Fig. 1: New CERN irradiation facility (from[8])

\section{The Karlsruhe Proton Cyclotron}

The compact Cyclotron operated by ZAG Zyklotron AG on the Campus north of Karlsruhe Institute of Technology (KIT) accelerates protons up to $25 \mathrm{MeV}$ [12]. A proton 
beam with a typical current of $1.5 \mu \mathrm{A}$ is extracted to the target area with a beamspot size of 7 $\mathrm{mm}$ (FWHM). The total flux of protons at the target is $2.310^{13} \mathrm{ps}^{-1} \mathrm{~cm}^{-2}$. With a scanning procedure objects having a size up to $40 \mathrm{~cm}$ x $60 \mathrm{~cm}$ can be irradiated. Special care is devoted to cooling with nitrogen which can reduce the temperature in the insulated irradiation box down to $-40^{\circ} \mathrm{C}$. Cooling is particularly important at irradiation facilities with lower energies of charged particles, since the large ionization and thus stopping power can cause heating of the target sample and uncontrolled annealing of defects. Another consequence of the high stopping power is the non-negligible energy loss of protons in the beam. Since the damage factor depends on the proton energy, only thin targets (less than $1 \mathrm{~mm}$ ) can be irradiated or/and energy losses have to be taken into account. Dosimetry with nickel foil has an error of $12 \%$. The damage factor is 2.0 and the measurement of the equivalent fluence has an error of $20 \%$ [13].

\section{Cyclotron Research Centre (CRC) at Louvain}

The variable energy cyclotron at CRC is capable of accelerating protons up to $65 \mathrm{MeV}$ [14]. There are several areas with beams of protons, neutrons and heavy ions. The neutron Irradiation facility produces neutrons from a $50 \mathrm{MeV}$ deuteron beam impinging on a $\mathrm{Be}$ target. The neutrons have a continuous spectrum up to $50 \mathrm{MeV}$ with a mean energy of 20 $\mathrm{MeV}$. The beam with diameter of approximately $4 \mathrm{~cm}$ can have a flux of $7.310^{10} \mathrm{nscm}^{-2}$. A cryogenic box capable of cooling the device under test (DUT) down to $-20^{\circ} \mathrm{C}$ with dry air during the whole process of irradiation and deactivation is available [15]. The light ion irradiation facility provides beam of protons with energies between 20 and $65 \mathrm{MeV}$. The beam is extracted through a $0.2 \mathrm{~mm}$ thin copper window which serves also as a diffuser. Samples are placed behind collimators. The uniformity of the beam with a maximum flux of $510^{8} \mathrm{ps}^{-1} \mathrm{~cm}^{-2}$ and approx. $8 \mathrm{~cm}$ diameter, is better than $10 \%$.

\section{Birmingham MC40 Cyclotron}

The cyclotron accelerates beams of protons (3-40 MeV), deuterons (5.5-20 MeV), 3He (9-53 MeV) and $4 \mathrm{He}(11-40 \mathrm{MeV})$. Beam currents up to $50 \mu \mathrm{A}$ (hydrogen) or $20 \mu \mathrm{A}$ (helium) are routinely available [16]. A new irradiation facility with $27 \mathrm{MeV}$ protons has been commissioned recently. For irradiation purposes the beam is collimated to $1 \mathrm{~cm}^{2}$ with vertical and horizontal plates. At this size a $10^{15} \mathrm{ncm}^{-2} 1 \mathrm{MeV}$ neutron equivalent fluence can be reached in $80 \mathrm{~s}$. The scanning area is limited to the window of $80 \mathrm{~mm}$ x $140 \mathrm{~mm}$ with 10 $\%$ uniformity. First irradiations of silicon sensors using an air cooling system and a glycol heat exchanger $\left(-10^{\circ} \mathrm{C}\right)$ showed evidence of overheating of samples and annealing of damage during irradiation. Therefore a new system with evaporative cooling by dripping a controlled quantity of liquid $\mathrm{N}_{2}$ on a metal box was developed and the first results were successful. The temperature of samples heated with $1 \mu \mathrm{A}$ beam was kept below $-5^{\circ} \mathrm{C}$, low enough to prevent annealing during the irradiation.

\section{Triga Nucler Reactor at Jožef Stefan Institute.}

Irradiations at Triga Mark II $[17,18]$ are done by insertion of samples directly into the reactor core (Fig. 2). The spectrum of neutrons covers energies from thermal to several MeV. Only fast neutrons with energies larger than $100 \mathrm{keV}$ contribute significantly to the NIEL in silicon, the contribution of slower (thermal, epithermal) is only about $1 \%$. The damage 
constant for fast neutrons was measured to be $0.90 \pm 0.05$ [2] which is in excellent agreement with the value of $0.88 \pm 0.05$ [19] calculated from theoretical predictions of neutron NIEL [20]. The size of samples is limited by the size of tubes which lead to the core. A small tube allows irradiations of samples with a maximum diameter of $25 \mathrm{~mm}$ while an elliptic tube has inner diameter of $7 \mathrm{~cm}$ and $5 \mathrm{~cm}$. The length of samples is limited by the size of the core to $15 \mathrm{~cm}$. The flux of fast neutrons $\left(3.310^{12} \mathrm{ncm}^{-2} \mathrm{~s}^{-1}\right.$ at full power of $\left.250 \mathrm{~kW}\right)$ can be tuned by reducing the power to the one Watt scale. The environmental temperature inside the core is $20^{\circ} \mathrm{C}$. The temperature of samples increases to $45^{\circ} \mathrm{C}$ during irradiation which keeps annealing low since irradiation times are short compared to annealing times of defects in silicon. A fluence of $10^{16} \mathrm{ncm}^{-2}$ can be reached in less than one hour.

Larger samples (few $\mathrm{dm}^{2}$ ) can be irradiated outside the reactor core, where fluxes up to $10^{7} \mathrm{ncm}^{-2} \mathrm{~s}^{-1}$ can be used.

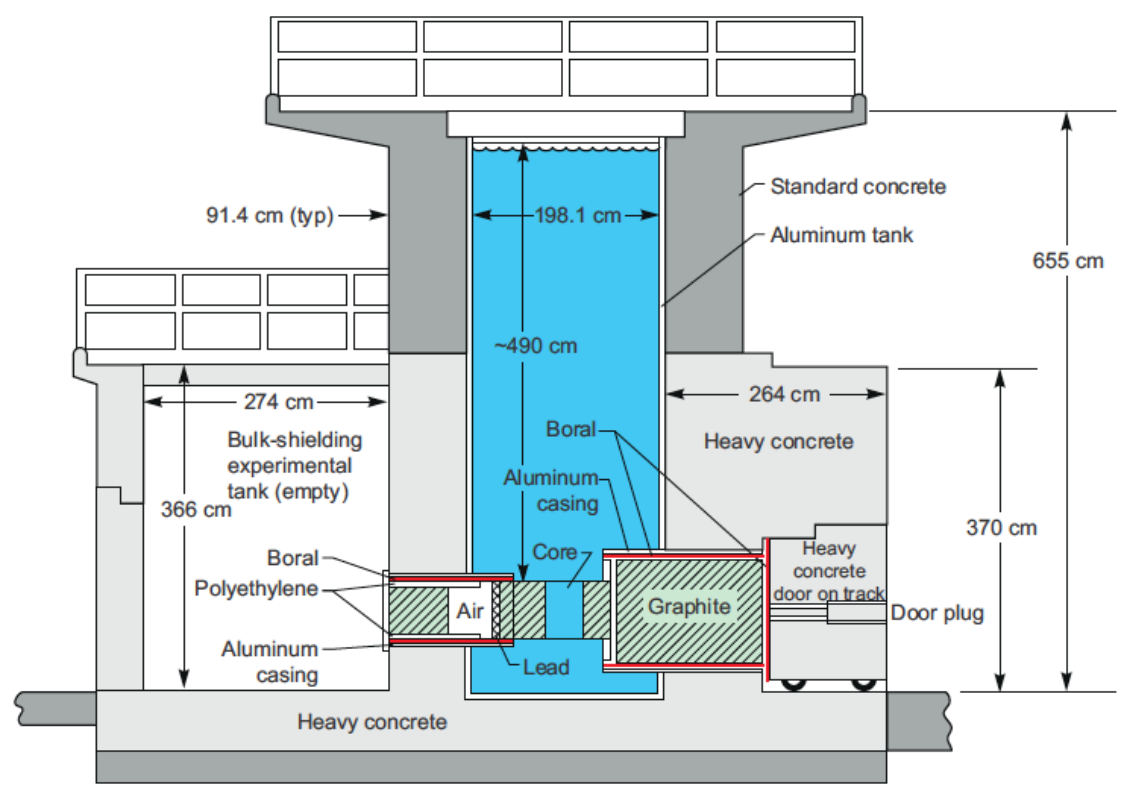

Fig. 2: TRIGA reactor at JSI, side view (from [17])

\section{Cyclotron on PSI Villigen}

$300 \mathrm{MeVc}^{-1}$ pions having an energy of $191.3 \mathrm{MeV}$ are available in the irradiation beam at the Paul Scherrer Institute Cyclotron [21]. They are produced with a beam of $590 \mathrm{MeV}$ protons impinging on secondary target. The flux and beam size depend on the position along beam line an are listed in Table I. The damage factor was measured to be $1.5 \pm 0.3$ [22].

\begin{tabular}{|c|c|c|c|}
\hline $\begin{array}{c}\text { Position } \\
\text { along the } \\
\text { beam line }\end{array}$ & Beam size & Beam size & \\
\hline $\mathrm{z}$ & $\mathrm{x}$ & $\mathrm{y}$ & Flux \\
\hline$(\mathrm{mm})$ & $\begin{array}{c}\text { FWHM } \\
(\mathrm{mm})\end{array}$ & $\begin{array}{c}\text { FWHM } \\
(\mathrm{mm})\end{array}$ & $\left(10^{14} \mathrm{~cm}^{-2} \mathrm{day}^{-1}\right)$ \\
\hline 50 & 16 & 13 & 1.5 \\
\hline 100 & 17 & 16 & 1 \\
\hline 150 & 18 & 23 & 0.5 \\
\hline 200 & 21 & 30 & 0.4 \\
\hline 250 & 24 & 38 & 0.25 \\
\hline
\end{tabular}

Table I. Pion beam characteristics at PSI 


\section{Summary}

There are other facilities around the word that are used for irradiations, for example the Los Alamos National Laboratory [23] provides $800 \mathrm{MeV}$ protons with a flux up to $510^{11}$ $\mathrm{pcm}^{2} \mathrm{~s}^{-1}$ and CYRIC in Japan provides a beam of $70 \mathrm{MeV}$ protons [24]. An overview of facilities is given in Table II. Access to these facilities is particularly important for the future development of radiation hard detectors. Therefore the European commission co-funded the transnational access to several facilities within Framework Programme 7 Capacities, Grant Agreement 262025 (AIDA).

\begin{tabular}{|c|c|c|c|c|c|c|}
\hline Institution & Facility & Source & Particles & $\begin{array}{l}\text { Energy } \\
(\mathrm{MeV})\end{array}$ & $\begin{array}{c}\text { Max. Flux } \\
\mathrm{cm}^{-2} \mathrm{~s}^{-1}\end{array}$ & $\begin{array}{c}\text { AIDA } \\
2011-2015\end{array}$ \\
\hline CERN & IRRAD & PS & $p$ & 24000 & $210^{11}$ & $\checkmark$ \\
\hline KIT & $\begin{array}{l}\text { Compact Cyclo- } \\
\text { tron }\end{array}$ & Cyclotron & $p$ & 25 & $210^{13}$ & $\checkmark$ \\
\hline $\mathrm{UCL}$ & $\mathrm{NIF}$ & Cyclotron & $\mathrm{n}$ & $<50$ & $710^{10}$ & $\checkmark$ \\
\hline UCL & LIF & Cyclotron & $p$ & $20-65$ & $510^{8}$ & $\checkmark$ \\
\hline UoB & MC40 & Cyclotron & $p$ & 26 & $1.510^{13}$ & \\
\hline JSI & TRIGA MARK III & Reactor & $\mathrm{n}$ & $<15$ & $410^{12}$ & $\checkmark$ \\
\hline PSI & PIF & Cyclotron & pions & 191 & $10^{10}$ & \\
\hline LANL & LANSC Linac & Linac & $p$ & 800 & $510^{11}$ & \\
\hline CYRIC & CYRIC & Cyclotron & $p$ & 70 & & \\
\hline
\end{tabular}

Table II: List of irradiation facilities

\section{Acknowledgements}

The author would like to thank the many people that helped to collect the information summarized in this paper: M. Moll and F. Ravotti for the CERN irradiation facility, A. Dierlamm for Karlsruhe Proton Synchrotron, E. Cortina for CRC at Louvain and J. Wilson for Birmingham MC40 Cyclotron.

\section{References}

[1] Letter of Intent for the Phase-II Upgrade of the ATLAS Experiment, CERN-LHCC-2012-022 ; LHCCI-023

[2] M. Moll et al., Leakage current of hadron irradiated silicon detectors - material, dependence, NIM A 426, (1999) 87-93

[3] G. Lindstroem et al., Radiation hard silicon detectors - developments by the RD48 (ROSE) collaboration, NIM A 466 (2001) 308-326

[4] G. Kramberger et al., Effective traping time of electrons and holes in different silicon materials irradiated with neutrons, protons and pions, NIM A 481 (2002) 297 - 305

[5] A. Ruzin et al., Comparison of Radiation Damage in Silicon Induced by Proton and Neutron Irradiation, IEE TNS, vol. 46., No.5, (1999) 1310 - 1313 
detectors, NIM A 583 (2007) 104-108

[7] https://irradiation.web.cern.ch/irradiation

[8] F. Ravotti: CERN-PH Irradiation facilities, PH-ESE Electronics Seminars, CERN, June 2014

[9] B. Gkotse et al., A New High-Intensity Proton Irradiation Facility at the CERN PS East Area, Presentation at TIPP 2014, Amsterdam, 2-6 June 2014

[10] M. Moll et al., Relation between microscopic defects and macroscopic changes in silicon detector properties after hadron irradiation, NIM B 186 (2002) 100-110

[11] http://www.cern.ch/charm

[12] http://www.ekp.kit.edu/english/irradiation_center.php

[13] A. Dierlamm, Irradiations in Karlsruhe, presentation at 16th RD50 Workshop on Radiation hard semiconductor devices for very high luminosity colliders, Barcelona, 31 May-2 June 2010,

[14] http://www.cyc.ucl.ac.be/index.php

[15] G. Berger, AIDA second Annual report, UCL, Belgium, presentation at AIDA 2nd Annual MeetingLNF Frascatti April 102013

[16] K. Parker et al., Irradiation of ATLAS sensors and materials to HL-LHC fluences using the Birmingham Cyclotron Facility, Presentation at TIPP 2014, Beurs van Berlage, 2-6 June 2014

[17] L. Snoj et al. Computational analysis of irradiation facilities at the JSI TRIGA reactor, Applied Radiation and isotopes 70 (2012) 483-488

[18] http://www-f9.ijs.si/ mandic/ReacSetup.html

[19] D. Žontar et al., Time development and ßux dependence of neutron-irradiationinduced defects in silicon pad detectors, NIM A 426 (1999) 51-55

[20] A.M. Ougoag et al., Differential displacement kerma cross sections for neutron interactions in Si and GaAs, IEEE Trans. Nucl. Sci. NS-37 (1990) 2219-2228

[21] http://pif.web.psi.ch/

[22] C. Buttar et al., Si detector macroscopic damage parameters during irradiation from measurements of dark current evolution with fluence, Nuclear Science Symposium Conference Record (2002) 624-627

[23] http://wnr.lanl.gov/

[24] N. Unno, et. al, p-bulk silicon microstrip sensors and irradiation, NIMA 579 (2007) 614-622 